\title{
Teledentistry for screening orthodontic patients
}

\author{
Teledentistry for screening new patient orthodontic referrals. Part 1: A randomised controlled trial. \\ N. A. Mandall, K. D. O'Brien, J. Brady, H. V. Worthington and L. Harvey Br Dent J 2005; 199: 659-662
}

\section{Objective}

The primary aim was to evaluate the validity of a teledentistry system for screening new patient orthodontic referrals. The secondary aims were to evaluate whether the teledentistry system affected i) referral rates ii) inappropriate referral rates iii) number of failed appointments. Study design

Randomised controlled trial

Sample

Fifteen dental practices in Greater Manchester, UK, were randomly allocated to either a teledentistry test group $(n=8)$ or a control group $(n=7)$. They referred 327 patients over a 15 -month period.

Method

Practitioners in the test group referred patients to one of two consultant orthodontists via a 'store and forward' teledentistry system consisting of photographs sent as email attachments. The decision to accept or not accept a referral on this basis was compared with the same decision choice when the same patient was subsequently seen on a new patient clinic. This measured the validity of the system with the clinic's decision used as the gold standard. Patients in the control group were referred using the usual letter system. Referral rates, inappropriate referrals and number of failed appointments were then compared between the teledentistry and control groups.

Results

The sensitivity (true positive value) of the teledentistry system was high at 0.80 with a positive predictive value of 0.92 . The specificity (true negative value) was slightly lower at 0.73 with a negative predictive value of 0.50 . The inappropriate referral rate for the teledentistry group was $8.2 \%$ and for the controls $26.2 \%(p=0.037)$. There was no statistically significant difference in clinic attendance between teledentistry and control groups ( $p=0.36)$.

Conclusions

Teledentistry is a valid system for positively identifying appropriate new patient orthodontic referrals. However, there is a risk that a patient is not accepted on the teledentistry system who would benefit from a full clinical examination. Teledentistry could be a significant factor in reducing the inappropriate referral rate. Patient participation in a teledentistry system does not appear to mean they are any more likely to attend their hospital appointment.

\section{IN BRIEF}

- Teledentistry for orthodontic referrals has been successfully used by eight GDP practices in Greater Manchester.

- The benefit to the GDP is availability of specialist orthodontic advice based on clinical photographs $+/$ - radiographs sent via email.

- The benefit to the patient is that they may be saved a hospital visit if their referral is first assessed via teledentistry. This will be particularly helpful if patients live far from the orthodontic provider.

\section{COMMENT}

Teledentistry has been advocated as a relatively quick and easy way for GDPs to obtain advice from an orthodontist, particularly for the timing of treatment and who should carry it out. The authors of this trial conclude that the store and forward emailed-based system for sharing dental records and photographs is an acceptable method of processing orthodontic referrals.

One interesting finding was that the number of 'inappropriate' orthodontic referrals was reduced among practitioners in the teledentistry group. The authors defined inappropriate as patients with poor oral hygiene, a mild malocclusion, who were too early for treatment, or other reasons, such as they did not want treatment.

It is difficult to see how teledentistry perse could be responsible for this, although it might be considered a useful outcome. One suggestion is that the teledentistry group were being more selective in their referrals, because of the time it took to take the records. This theory is supported by the fact that there were significantly more referrals from the control group during the trial compared with the experimental group.

It might be interesting to examine the number of referrals from the experimental group compared during a similar time period before the trial and see if it was lower. Another suggestion for the reduced number of inappropriate referrals was that the practitioners knew they were in a trial, which in itself influenced their behaviour (the so-called Hawthorne effect).

The use of the term 'inappropriate' might be a little harsh on the referring GDP. We know that children's tooth brushing habits can be erratic and criteria such as whether the malocclusion is too mild or too early, might be exactly the information the GDP is seeking. It could be argued that the widespread introduction of teledentistry might have the opposite effect to the results of this trial and lead to an increase in the number of referrals for advice about timing and appropriateness of treatment because the practitioner will have ready access to the orthodontist without inconveniencing the patient.

A slightly worrying outcome from the study was that approximately one in six patients who were deemed inappropriate referrals as a result of teledentistry were considered appropriate when examined clinically. The authors suggest that this might be due to poor oral hygiene or the perception of poor oral hygiene when using teledentistry. I would suggest that this requires further investigation.

Overall the study is a very useful contribution to the case for using modern communications technology to improve the service provided to patients. In the second part of this study the attitudes of general dental practitioners regarding the use of teledentistry for orthodontic referrals are explored.

P. Benson, Senior Lecturer in Orthodontics, School of Clinical Dentistry, Sheffield

doi: 10.1038/sj.bdj.4812932 